\title{
Parallel and Distributed Programming
}

\author{
Thilo Kielmann ${ }^{1}$, Andrea Clematis ${ }^{1}$, Sergei Gorlatch ${ }^{2}$, \\ and Alexey Lastovetsky ${ }^{2}$ \\ 1 Topic Chairs \\ 2 Members
}

Developing parallel or distributed applications is a hard task and it requires advanced algorithms, realistic modeling, efficient design tools, high-level programming abstractions, high-performance implementations, and experimental evaluation. Ongoing research in this field emphasizes the design and development of correct, high-performance, portable, and scalable parallel programs. Related to these central needs, important work addresses methods for reusability, performance prediction, large-scale deployment, self-adaptivity, and fault-tolerance. Given the rich history in this field, practical applicability of proposed methods, models, algorithms, or techniques is a key requirement for timely research. This topic is focusing on parallel and distributed programming in general, except for work specifically targeting multicore architectures, which has matured to becoming a Euro-Par topic of its own.

This year, 17 papers were submitted to this topic. Each submission was reviewed by at least four reviewers and, finally, we were able to select five regular papers, spanning the topic's scope, ranging from low-level issues like locking schemes and exceptions, all the way up to the parallelisation of a biocomputational simulation.

In particular, Dalessandro et al. propose "Transactional Mutex Locks", combining the generality of mutex locks with the scalability of software transactional memory. In "Exceptions for Algorithmic Skeletons", Leyton et al. describe how to handle exceptions without breaking the high-level abstractions of algorithmic skeletons. Emoto et al. contributed "Generators-of-generators Library with Optimization Capabilities in Fortress", a library for constructing parallel skeletons from nested data structures. In "User Transparent Task Parallel Multimedia Content Analysis", van Kessel et al. present a domain-specific, user-transparent programming model. Last but not least, Duta et al. exploit the algorithmic parallelism in biocomputational simulations in their paper "Parallel Simulation for Parameter Estimation of Optical Tissue Properties".

We are proud of the scientific program that we managed to assemble. Of course, this was only possible by combining the efforts of many. We would like to take the opportunity to thank the authors who submitted their contributions, and the external referees who have made the scientific selection process possible in the first place. 